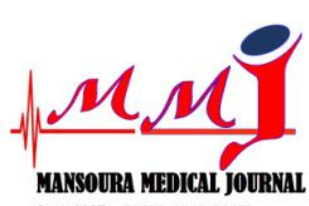

\author{
Mansoura Medical Journal \\ (Official Journal of Mansoura Faculty of Medicine) \\ pISSN: 1110-211X; eISSN: 2735-3990
}

\title{
Histological Study of Hyaline Articular Cartilage changes in a Rat Model of Complete Freund's Adjuvant-Induced arthritis of the Knee Joint
}

\author{
Sara Al-Sherief, Azza El- Hadidy, Shereen Hamed, Amany El-Hawwary, Shireen Mazroa \\ Department of Medical Histology and Cell Biology, Faculty of Medicine, Mansoura University, Egypt
}

DOI: $10.21608 / \mathrm{mjmu} .2021 .98112 .1042$

\section{Submit Date:}

Accept Date:

Available online:

Keywords

- Arthritis

- knee joint

- Animal model

- Rat

- Complete Freund's Adjuvant

\section{Abstract}

Background: Arthritis is the most common joint disorder. The most significant structural change seen in arthritis is the degeneration of the articular cartilage. Numerous animal models are used to reproduce arthritis. In this paper, we assessed the ability of complete Freund's adjuvant (CFA) to induce degenerative changes of hyaline articular cartilage in rat chronic arthritis model. Methods: In this study, 42 adult male albino rats were used, divided into: control group I (18 rats) and arthritis group II (24 rats). Arthritis was induced unilaterally by a single intra-articular injection of $125 \mu \mathrm{l}$ of CFA in the right knee joint of each rat. The samples were collected 2, 6, and 10 weeks post-injection. Hematoxylin \& Eosin (H\&E) stain, Masson's trichrome stain, Safranin O (S.O) stain were used to assess articular cartilage degeneration during inflammation.Results: The articular cartilage of rats of arthritis group II showed progressive inflammatory cell infiltration in the synovial membrane. Chondrocytes showed degeneration, hypertrophy and extensive proliferation. Moreover, the matrix exhibited fibrillations, fissuring, delamination and denudation with fibrous tissue within the denuded surface. The thickness of the articular cartilage, the collagen content and the proteoglycan content showed significant decrease in rats sacrificed after 10 weeks compared to rats sacrificed after 2 weeks and 6 weeks from the onset of the experiment.Conclusions: Intra-articular injection of complete Freund's adjuvant induces progressive degeneration of the hyaline articular cartilage of the knee joint of rats. It can be useful for studies on mechanisms and strategy of treatment of knee joint arthritis. 


\section{INTRODUCTION}

The knee joint is the most complicated and largest joint in the body. The articulating surfaces of bones of the knee are all coated with articular cartilage. The function of articular cartilage is to absorb shock, facilitate motion and decrease the stress occurring during joint movement. However, this joint is more susceptible to physical attacks because it bears huge weight (Hirschmann and Müller, 2015; Wu, 2015; Ondrésik et al., 2016).

Arthritis is the most common joint disorder. It is considered as a fundamental economic burden. The most significant structural change seen in arthritis is the degeneration of the articular cartilage. Several animal models have been developed to study the pathogenesis of arthritis and the efficacy of new diagnostic tools and therapeutic interventions. To date, there is no single ideal experimental model that permits investigation of all features of arthritis, and each model of induction has distinct advantages and disadvantages. These models can be divided into spontaneous, surgically induced and chemically induced models (Hunter \& Felson, 2006; Little \& Smith, 2008 Evans, 2013; Nickien et al., 2018; Harrell et al., 2019).

Spontaneous arthritis occurs in various strains of mice, guinea pigs and macaques. These animals are considered predisposed to developing spontaneous idiopathic arthritis, however there is concern that the underlying mechanism for the particular strain of animal developing the disease may not necessary be the same as for arthritis in humans. Another disadvantage of using the spontaneous arthritis models is the more variable and extended time frame of disease progress, particularly for the larger animals (Quasnichka et al., 2006).

Surgically induced destabilization of joints by anterior cruciate ligament transection or meniscal injury is a common method used for induction of arthritis. An advantage of this model is temporal control of disease induction and these models follow a predictable progressive disease onset. There are, however, limited clinical outcome measures currently available in many of the species used (Little \& Smith, 2008).

Chemically induced models of arthritis means induction of arthritis by intra-articular injection of agents, including collagenase, monosodium iodoacetate (MIA) and complete Freund's adjuvant (CFA). These models eliminate the need for surgery and the possibility of associated infection in some models. They are easy to induce and reproduce, and are useful for short-term studies. Administration of different dosages and intervals of compounds allows the study of various stages of disease development. For example, in rats, intraarticular injection of CFA, an inactivated dried mycobacteria which is responsible for stimulation of cell-mediated immunity that ultimately increases the production of certain immunoglobulins, induces arthritic changes in hyaline articular cartilage (Malfait et al., 2013; Tuncel et al., 2016; Mahdi et al., 2018).

The advantages and disadvantages of CFA arthritis model still need more research to assess the validity of this model for pre-clinical researches. So, the aim of this study was to assess the degenerative changes of articular cartilage in rat chronic arthritis model induced by complete Freund's adjuvant (CFA). 


\section{Materials and methods:}

This study was approved by Institutional Review Board (IRB), faculty of medicine, Mansoura University. Code number: MDP. 18. 06. 07. All the steps took place in Mansoura Experimental Research Center (MERC), faculty of medicine, Mansoura University.

\section{Experimental Animals and Design:}

Forty two adult male albino rats (90-110 days old, average weight of 150-200 g) were obtained and housed in plastic cages (4 rats per cage) on sawdust with free access to food and water adlibitum and kept at a controlled temperature of 22 $\pm 1{ }^{\circ} \mathrm{c}$, at $50 \%$ relative humidity, with 12 hour light I dark cycle for one week before the experiment.

Rats were divided randomly into group I (control group) which included 18 rats, and group II (arthritis group) which included 24 rats. Arthritis was induced unilaterally by a single intra-articular injection of $125 \mu \mathrm{l}$ of CFA (Sigma- Aldrich Company, Germany, Cat. No: F5881) in the right knee joint of each rat. The choice of CFA dose was based on Liu et al (2017) who used CFA in a dose of $100 \mu 1$ into the left hind limb to induce arthritis model and based on our pilot experiments in which lower doses were insufficient to induce sustained inflammation. Six animals from groups I and eight animals from group II were euthanized by inhalation of Halothane (5\%) (Valentim et al., 2016) and knee joint samples were dissected and prepared for histological study after 2, 6 and 10 weeks from the onset of the experiment.

\section{Histological analysis:}

Paraffin para-sagittal (antero-posterior) sections of $5 \mu \mathrm{m}$ thickness passing through tibio-femoral articulation were prepared from the specimens. These sections were stained with haematoxylin and eosin stain for routine histological examination (Bancroft and Layton, 2013), Safranin O stain to determine proteoglycan content in articular cartilage (Kahveci et al., 2000) and Masson's trichrome staining for the demonstration of collagen fibers in articular cartilage (Bancroft and Layton, 2013).

N.B: The severity of the osteoarthritic process was assessed by OARSI grading method depending on microscopic changes (Pritzker et al., 2006):

\begin{tabular}{|c|c|}
\hline \multirow{2}{*}{$\begin{array}{l}\text { Grade 0: surface intact, cartilage } \\
\text { morphology intact }\end{array}$} & Matrix: normal architecture \\
\hline & Cells: intact, appropriate orientation \\
\hline \multirow{3}{*}{ Grade 1: surface intact } & $\begin{array}{l}\text { Matrix: superficial zone intact, oedema and/or superficial fibrillation (abrasion), focal } \\
\text { superficial matrix condensation }\end{array}$ \\
\hline & Cells: death, proliferation (clusters), hypertrophy \\
\hline & superficial zone reaction must be more than superficial fibrillation only \\
\hline \multirow{6}{*}{ Grade 2: surface discontinuity } & As above \\
\hline & +Matrix discontinuity at superficial zone (deep fibrillation) \\
\hline & \pm Cationic stain matrix depletion (Safranin O or Toluidine Blue) upper $1 / 3$ of cartilage \\
\hline & \pm Focal perichondronal increased stain (mid zone) \\
\hline & \pm Disorientation of chondron columns \\
\hline & Cells: death, proliferation (clusters), hypertrophy \\
\hline \multirow{5}{*}{ Grade 3: vertical fissures (clefts) } & As above \\
\hline & Matrix vertical fissures into mid zone, branched fissures \\
\hline & $\begin{array}{l} \pm \text { Cationic stain depletion (Safranin O or Toluidine Blue) into lower 2/3 of cartilage } \\
\text { (deep zone) }\end{array}$ \\
\hline & \pm New collagen formation (polarized light microscopy, Picro Sirius Red stain) \\
\hline & $\begin{array}{l}\text { Cells: death, regeneration (clusters), hypertrophy, cartilage domains adjacent to } \\
\text { fissures }\end{array}$ \\
\hline \multirow{2}{*}{ Grade 4: erosion } & Cartilage matrix loss: delamination of superficial layer, mid layer cyst formation \\
\hline & Excavation: matrix loss superficial layer and mid zone \\
\hline Grade 5: denudation & $\begin{array}{l}\text { Surface: sclerotic bone or reparative tissue including fibrocartilage within denuded } \\
\text { surface. Microfracture with repair limited to bone surface }\end{array}$ \\
\hline Grade 6: deformation & $\begin{array}{l}\text { Bone remodeling (more than osteophyte formation only). Includes: microfracture with } \\
\text { fibrocartilaginous and osseous repair extending above the previous surface }\end{array}$ \\
\hline
\end{tabular}




\section{Statistical analysis:}

Statistical parameters assessed in the study included:

1- Thickness $(\mu \mathrm{m})$ of hyaline articular cartilage covering the central part of femoral condyle in sections stained with $H \& E$ at a magnification $\mathrm{x} 40$.

2- Percentage $(\%)$ of area of collagen stained with Masson's trichrome stain in the hyaline articular cartilage covering femoral condyle at a magnification $\mathrm{x} 100$.

3- Percentage (\%) of area of proteoglycans stained with Safranin stain in the hyaline articular cartilage covering femoral condyle at a magnification $\mathrm{x} 100$.

For assessment of the previous parameters, at least 6 fields from all animals in each group of the experiment were randomly chosen for the examination and were photographed in department of medical histology and cell biology, faculty of medicine, Mansoura university، using Olympus ${ }^{\circledR}$ digital camera installed on Olympus ${ }^{\circledR}$ microscope with $0.5 \mathrm{X}$ photo adaptor, using objective lens depending on the required analysis. The result images were analyzed on Intel ${ }^{\circledR}$ Core $\mathrm{I} 7 \AA$ based computer using Video Test Morphology ${ }^{\circledR}$ software (Russia). The data were tabulated, coded then analyzed using the computer program SPSS software program. In the statistical comparison between the different groups, the significance of difference was tested using ANOVA (One-Way Analysis of Variance) to compare between more than two groups of numerical (parametric) data followed by post-hoc tukey for multiple comparisons. Significance was realized at $\mathrm{P}$ value $<0.05$. All the data were represented in tables and histograms (Hazra and Gogtay, 2016).

\section{Results:}

\section{A) Results of histological study:}

\section{Group I (control group):}

- H\&E stained sections of this group revealed that the hyaline articular cartilage covering both femoral condyle and tibial plateau had a smooth surface and consisted of chondrocytes surrounded by extracellular matrix (ECM). The subchondral bone was formed of bone trabeculae and bone marrow spaces. The junction between the hyaline articular cartilage and the subchondral bone was wavy (Figure 1). The chondrocytes and surrounding matrix were arranged in well ordered, appropriately oriented zones: the superficial zone, the middle zone, the deep zone and the calcified zone. The Superficial zone was the thinnest zone and the chondrocytes were flattened, densely packed and parallel to the surface. The middle zone was relatively thicker and the chondrocytes were slightly larger, more spherical in shape, forming isogenous groups. The chondrocytes in the deep zone were large in size and formed columns perpendicular to the articular surface. The tidemark appeared as a distinct basophilic thin line separating the deep zone from the calcified zone of cartilage. In the calcified zone, the chondrocytes were fewer in number and large in size with large lacunae (Figure 2).

- Masson's trichrome stained sections revealed the intense blue stained collagen in the matrix of the articular cartilage. Near the tidemark and in the deep zone, collagen was arranged as coarse bundles perpendicular to the surface (Figure 3).

- Safranin $\mathbf{O}$ / fast green stained sections showed that the proteoglycan (PG) content in the articular cartilage was stained red. The proteoglycan staining in the middle zone was more intense compared to the superficial zone, the deep zone and the calcified zones (Figure 4). 
- This group was considered of grade $\mathbf{0}$ according

to (OARSI) grading of arthritis because the matrix exhibited normal architecture and the cells were intact with appropriate orientation.

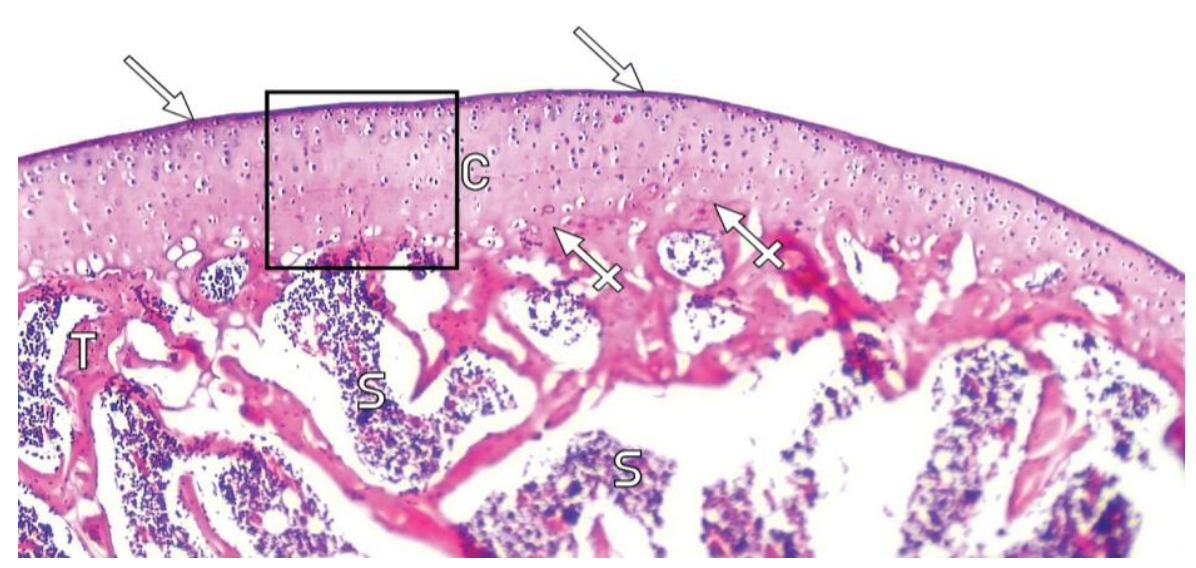

Figure (1): A photomicrograph of a para-sagittal section of control rat knee joint showing the hyaline articular cartilage (C) with smooth surface of cartilage (arrows). The junction between the hyaline articular cartilage and the subchondral bone appears wavy (crossed arrows). Note that the subchondral bone is formed of bone trabeculae (T) and bone marrow spaces (S).

(H\&E X 100)

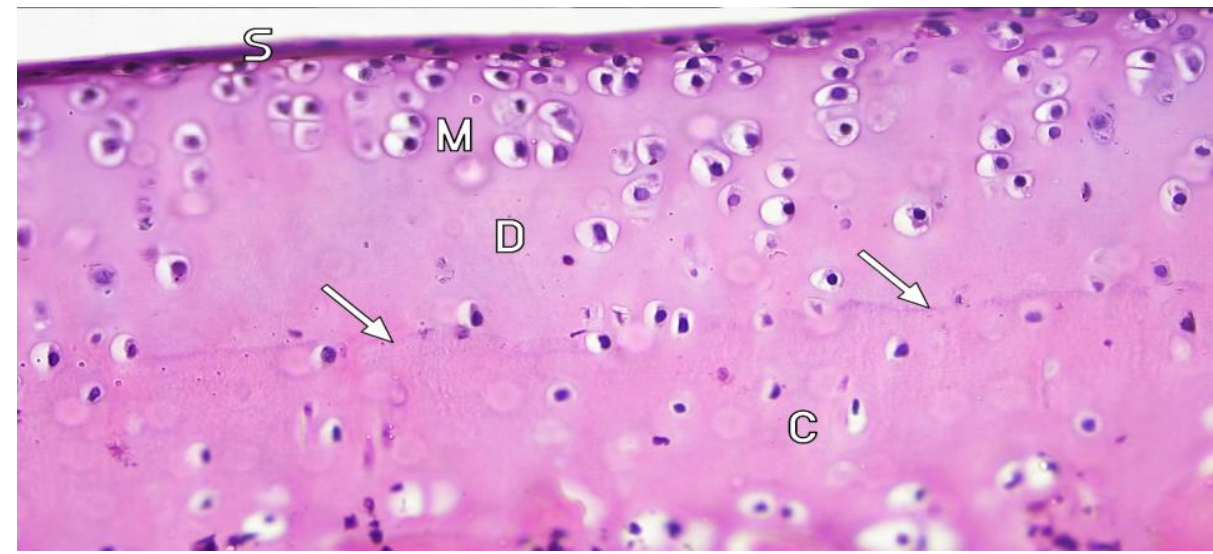

Figure (2): A high magnification of figure (1) showing that the chondrocytes and surrounding matrix are arranged in well oriented zones: the superficial zone (S), the middle zone (M), the deep zone (D) and the calcified zone (C). The tidemark (arrows) appears as intact, distinct basophilic thin line separating the deep zone from the calcified zone of cartilage. Note that chondrocytes vary in size, shape and number in each zone of the articular cartilage.

(H\&E x 400)

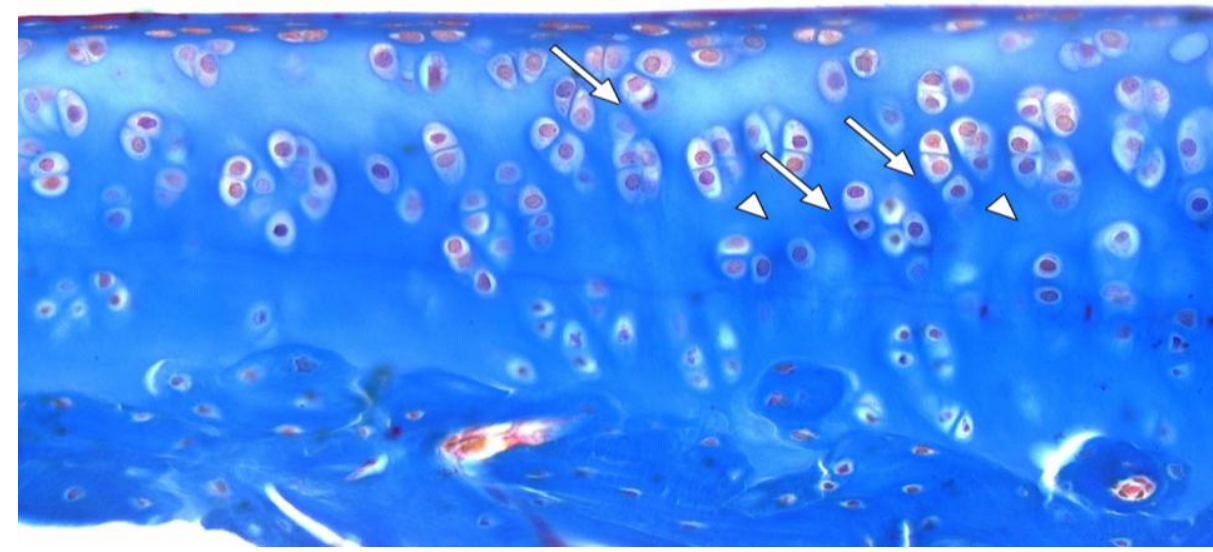

Figure (3): A photomicrograph of a para-sagittal section of control rat knee joint showing intense blue staining of collagen (arrow heads) in the matrix of the articular cartilage. In the deep zone, collagen is arranged as coarse bundles perpendicular to the surface (arrows) 


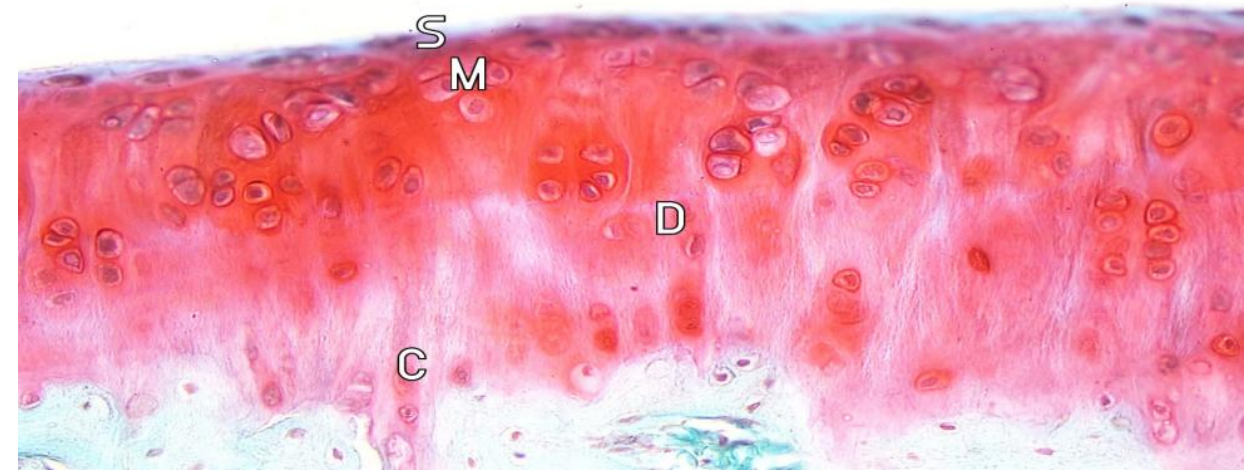

Figure (4): A photomicrograph of a para-sagittal section of control rat knee joint showing minimal red staining of proteoglycan at the superficial zone $(\mathrm{S})$. The middle zone $(\mathrm{M})$ shows the highest intensity of proteoglycan staining. In deep zone (D) and calcified zone (C), proteoglycan staining is less intense compared to the middle zone.

\section{Group II (arthritis group):}

a) Two weeks after CFA injection in the knee joint:

- H\&E stained sections revealed mild decrease in thickness of the articular cartilage as compared to control group. The surface of articular cartilage appeared smooth and continuous. The junction between articular cartilage and subchondral bone appeared flattened with absence of underlying bone trabeculae leaving wide bone marrow spaces. Extensive proliferation and disorganization of chondrocytes in different zones with disrupted tidemark integrity were noticed

(Figures 5, 6).

- Masson's trichrome stained sections and Safranin O/ fast green stained sections revealed a marked decrease in collagen and proteoglycans,

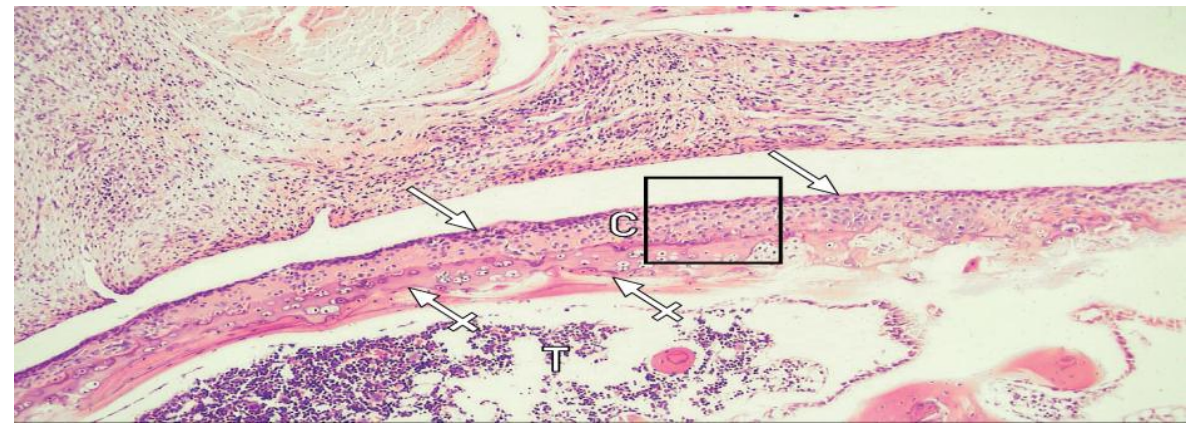

Figure (5): A photomicrograph of a para-sagittal section of knee joint from group II ( 2 weeks after CFA injection in the knee joint) showing the tibial plateau (T) covered with the hyaline articular cartilage (C). Notice a mild decrease in the thickness of articular cartilage and the surface is smooth and continuous (arrows). The junction between the articular cartilage and the subchondral bone is flattened (crossed arrows) with absence of underlying bone trabeculae leaving wide bone marrow spaces.
(Safranin $\mathbf{O}$ / fast green $\mathrm{x}$ 400)

respectively in the matrix of the articular cartilage particularly in superficial, middle and deep zones among heavy cell clusters. However, collagen and proteoglycans were moderately decreased in the calcified zone (Figures 7, 8).

- This group was considered of grade 2 according to (OARSI) grading of arthritis because the matrix exhibited marked decrease (depletion) in the upper part of cartilage and chondron columns were disoriented. In addition, the cells showed signs of proliferation (clusters), hypertrophy and degeneration. 


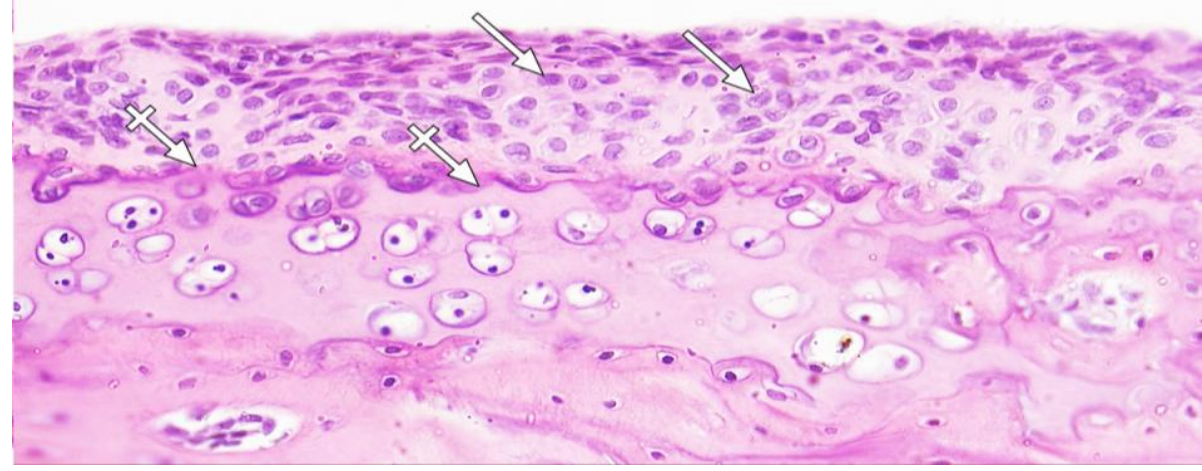

Figure (6): A high magnification of (figure 5) showing extensive proliferation and severe disorganization of the chondrocytes (arrows). Notice disrupted tidemark integrity (crossed arrows).

$(H \& E \times 400)$

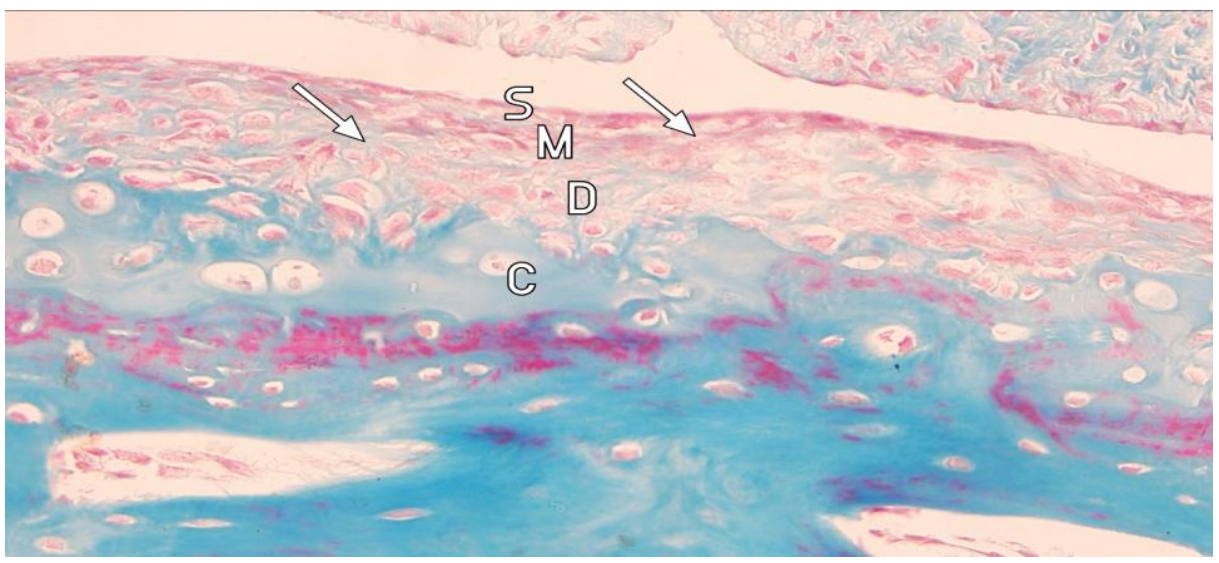

Figure (7): A photomicrograph of a para-sagittal section of knee joint from group II (2 weeks after CFA injection in the knee joint) showing a marked decrease in blue stained collagen in the matrix of the articular cartilage particularly in the superficial (s), middle (M) and deep (D) zones among heavy cell clusters (arrows). Notice that collagen in the calcified zone (C) is moderately decreased.

\section{(Masson's trichrome x 400)}

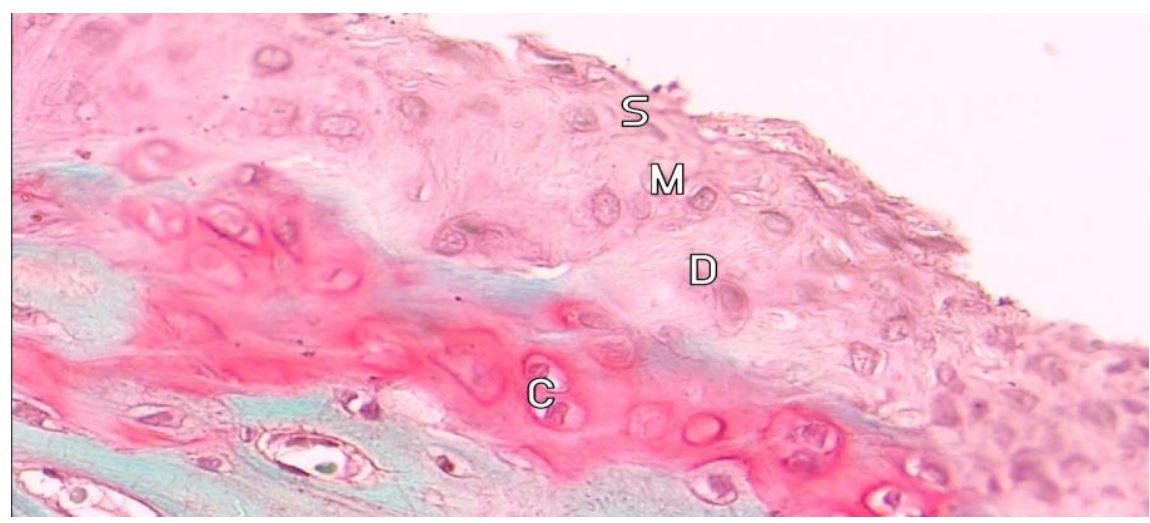

Figure (8): A photomicrograph of a para-sagittal section of knee joint from group II (2 weeks after CFA injection in the knee joint) showing a marked decrease of red stained proteoglycan content in the superficial (S), middle (M) and deep (D) zones, but in the calcified zone $(\mathrm{C})$, there is moderate decrease of proteoglycan content.

\section{(Safranin O/ fast green x 400)}

b) Six weeks after CFA injection in the knee joint:

- H\&E stained sections showed that the thickness of articular cartilage was obviously decreased in comparison to control and arthritis group after 2 weeks from the onset of the experiment. The articular cartilage showed transverse fissuring in the deep and calcified 
zones where the matrix was completely lost. In addition, there was delamination of the superficial and middle zones with separation from subchondral bone. Some areas of articular cartilage exhibited extensive chondrocyte proliferation in the form of heavy cell clusters, in addition to severe disorganization of chondrocytes and surrounding matrix with disrupted tidemark integrity (Figures 9, 10).

- Masson's trichrome stained sections and showed a marked decrease in collagen and proteoglycan content respectively among proliferating cell clusters with complete loss of collagen and proteoglycans in the region of transverse fissuring (Figures 11, 12).

- This group was considered as grade 4 according to (OARSI) grading of arthritis because the matrix exhibited fissuring and delamination. In addition, the cells showed signs of proliferation (clusters), hypertrophy and degeneration.

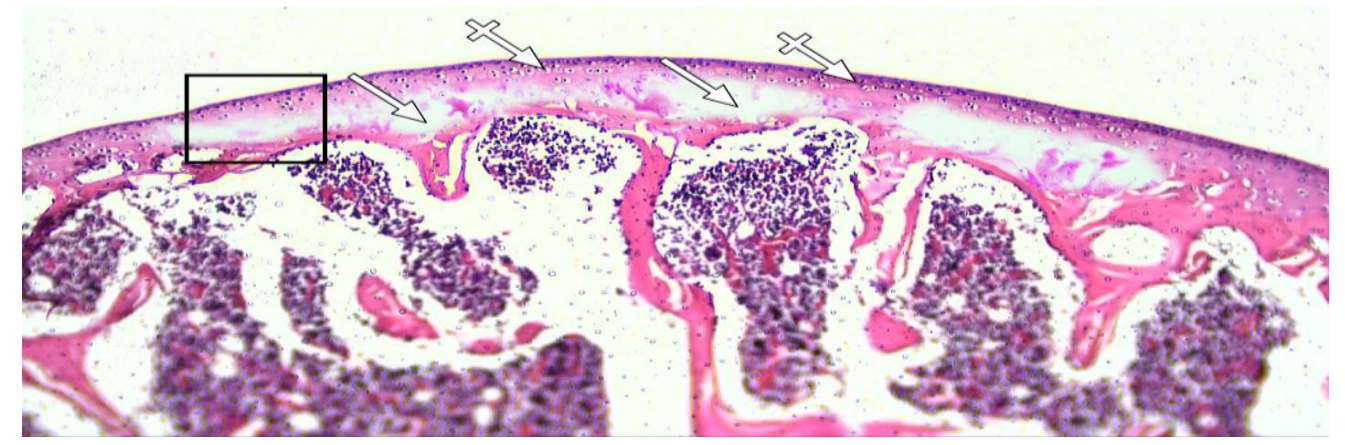

Figure (9): Para-sagittal section of knee joint from group II (6 weeks after CFA injection in the knee joint) showing obvious decrease in the thickness of articular cartilage in some areas. Notice areas of transverse fissuring (arrows) in which the matrix is completely lost. The superficial and middle zones are delaminated (crossed arrows) and separated from subchondral bone.

(H\&E X 100)

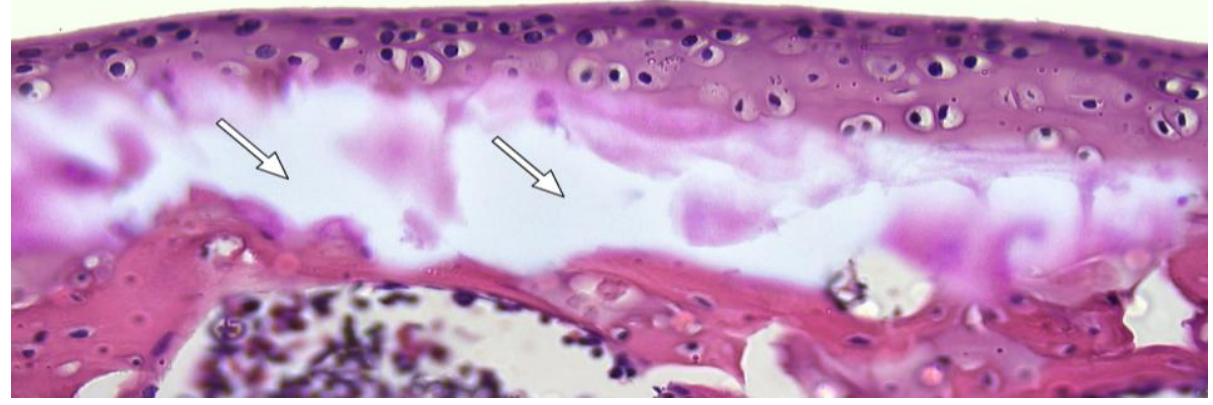

Figure (10): A high magnification of figure (9) showing area of transverse fissuring which is devoid of cells and matrix (arrows).

(H\&E X 400)

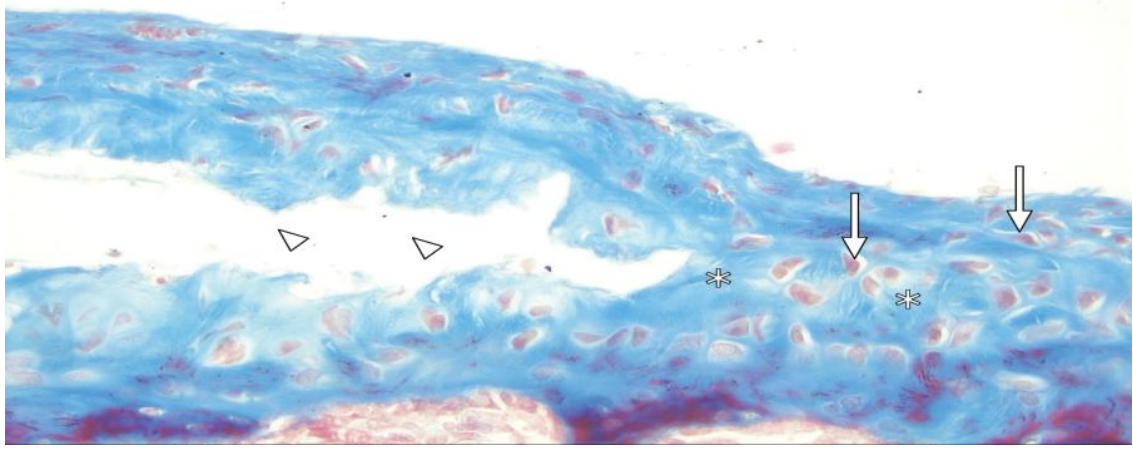

Figure (11): A photomicrograph of a para-sagittal section of knee joint from group II (6 weeks after CFA injection in the knee joint) showing marked decrease in collagen content (stars) among proliferating cell clusters (arrows). Notice complete loss of collagen in the region of transverse fissuring (arrow heads).

(Masson's trichrome x 400) 


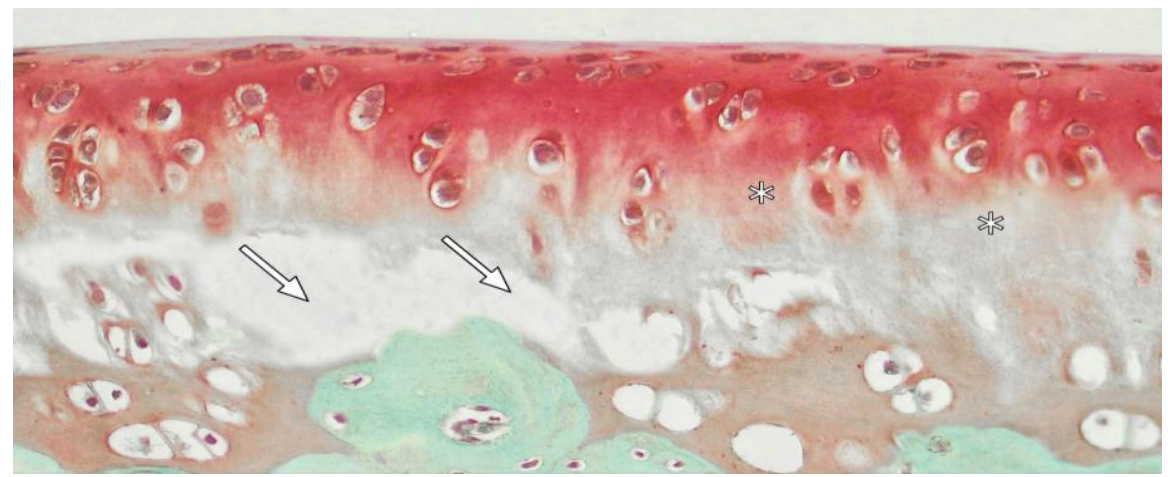

Figure (12): A photomicrograph of a para-sagittal section of knee joint from group II (6 weeks after CFA injection in the knee joint) showing a marked decrease in red stained proteoglycan content (stars) in articular cartilage with complete loss of proteoglycans in the region of transverse fissuring (arrows).

\section{c)Ten weeks after CFA injection in the knee} joint:

- H\&E stained sections revealed that there were areas of excavation in the articular cartilage where the matrix was completely lost in the superficial and mid zones. In addition, there were areas of denudation where articular cartilage was completely eroded to the level of bone. The denuded surface was covered with fibrous tissue. Deep fissures extending into the bone were noticed (Figures 13, 14, 15).

- Masson's trichrome stained sections and Safranin $\mathbf{O}$ / fast green stained sections revealed that there was a decrease of collagen and proteoglycan content, respectively. In some

\section{(Safranin $\mathrm{O}$ / fast green $\mathrm{x}$ 400)}

areas, the articular cartilage was replaced by fibrous tissue which contained collagen fibers and multiple nuclei (Figures 16, 17, 18, 19).

- This group was considered of grade 5 arthritis according to (OARSI) grading of arthritis because there were areas of denudation with reparative tissue "fibrous tissue" within the denuded surface.

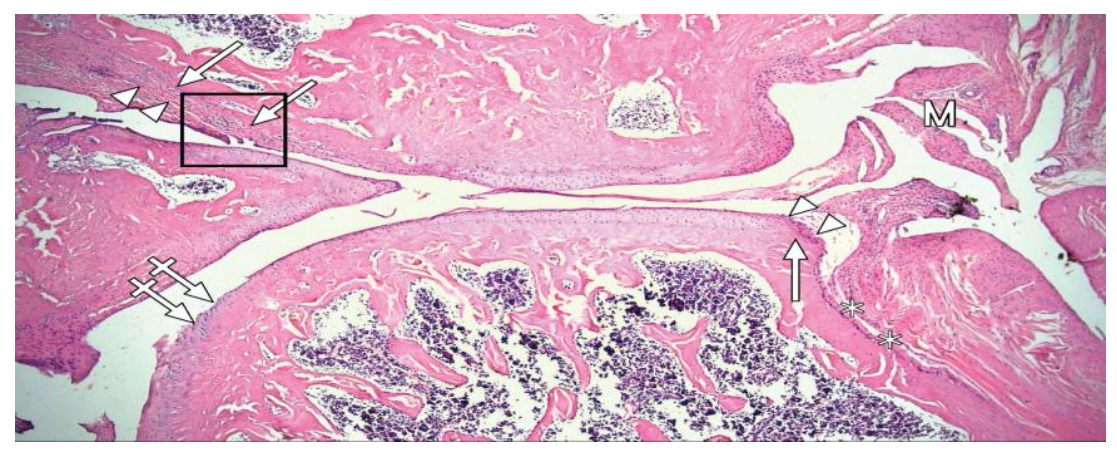

Figure (13): A photomicrograph of a para-sagittal section of knee joint from group II (10 weeks after CFA injection in the knee joint) showing area of excavation (crossed arrow) in the articular cartilage where the matrix is completely lost in the superficial and mid zones. In addition, there is area of denudation (arrows) where articular cartilage is completely eroded to the level of bone. The denuded surface is covered with fibrous tissue (arrow heads). Notice very deep fissure extending into the bone $(*)$. Notice eroded meniscus (M).

$($ H\&E X 40) 


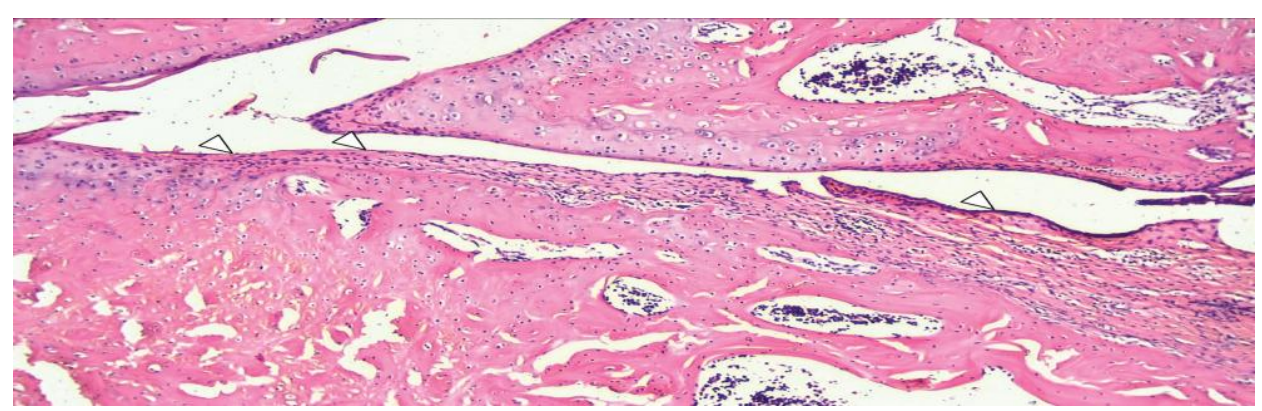

Figure (14): A high magnification of figure (13) showing fibrous tissue (arrow heads) covers the denuded surface.

(H\&EX 100)

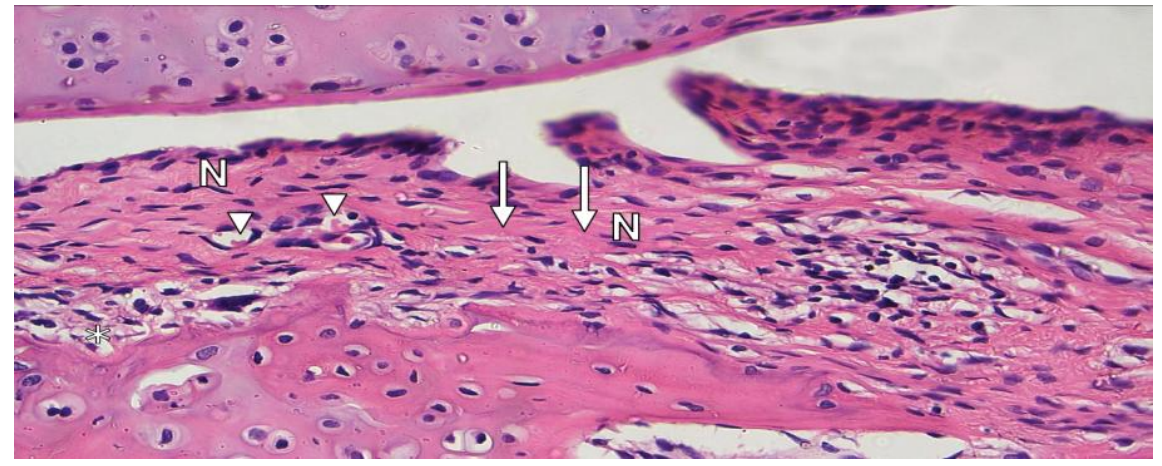

Figure (15): A high magnification of figure (13) showing the fibrous tissue which covers the denuded area and has multiple nuclei $(\mathrm{N})$, collagen fibers (arrows) in between and some blood vessels (arrow heads).

(H\&E X 400)

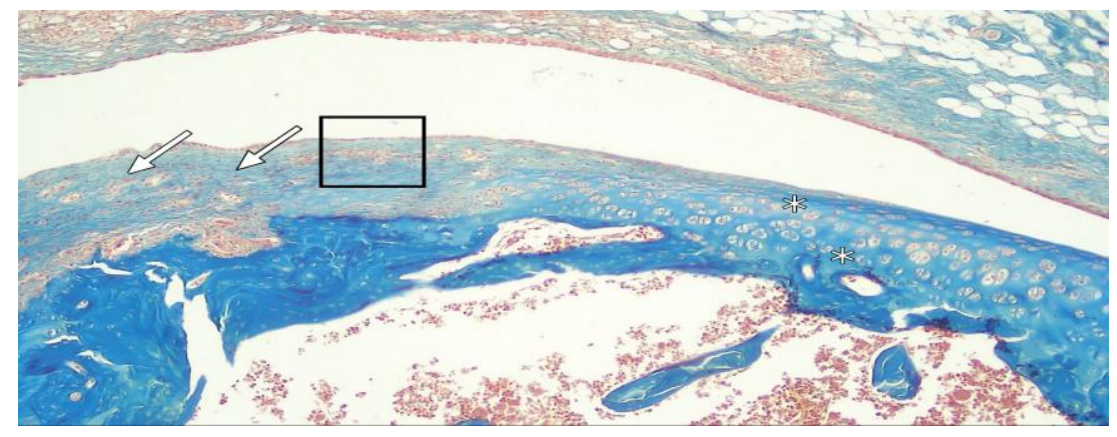

Figure (16): A photomicrograph of a para-sagittal section of the knee joint from group II (10 weeks after CFA injection in the knee joint) showing a marked decrease of collagen blue staining (stars) and in some areas, the articular cartilage is replaced by fibrous tissue (arrows).

(Masson's trichrome x 100)

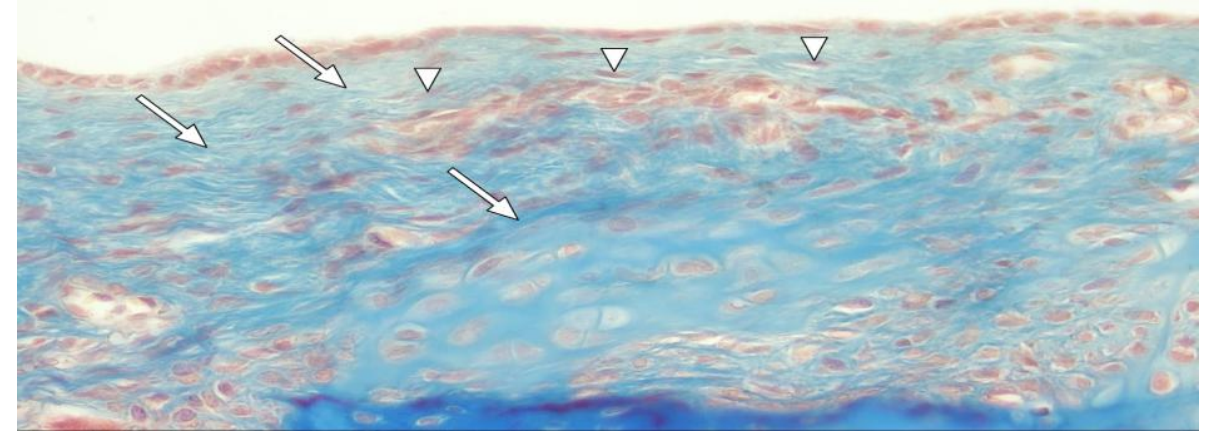

Figure (17): A high magnification of figure (16) showing that the articular cartilage is replaced by fibrous tissue which contains blue stained collagen fibers (arrows) and multiple nuclei (arrow heads). 


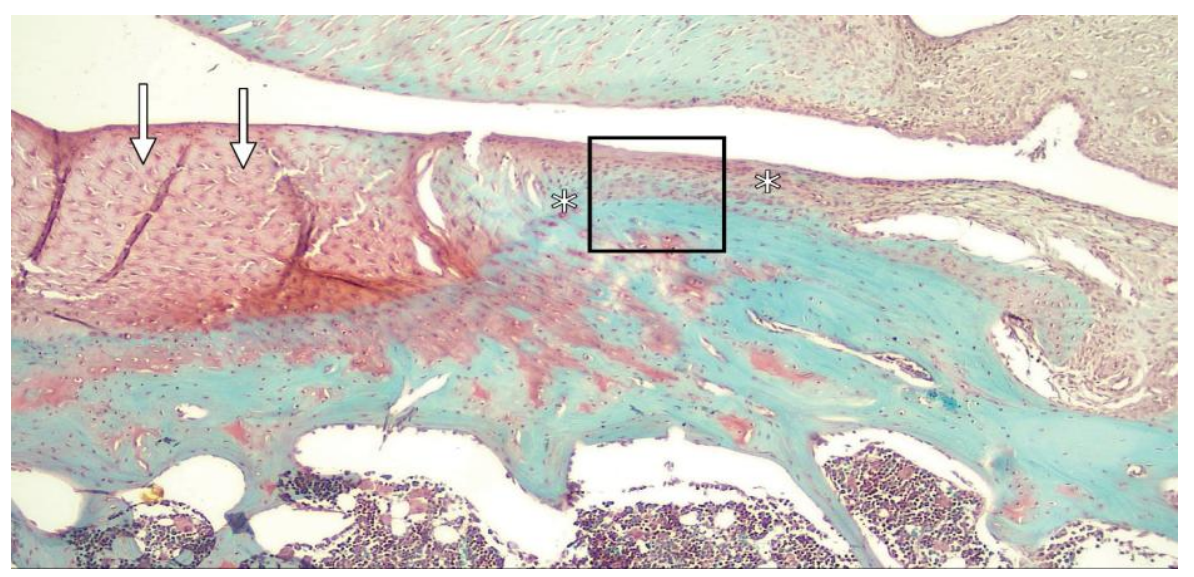

Figure (18): A photomicrograph of a para-sagittal section of the knee joint from group II (10 weeks after CFA injection in the knee joint) showing marked decrease of the proteoglycan red staining (stars) and in some areas, the articular cartilage is replaced by fibrous tissue (arrows).

(Safranin O/ fast green $x$ 100)

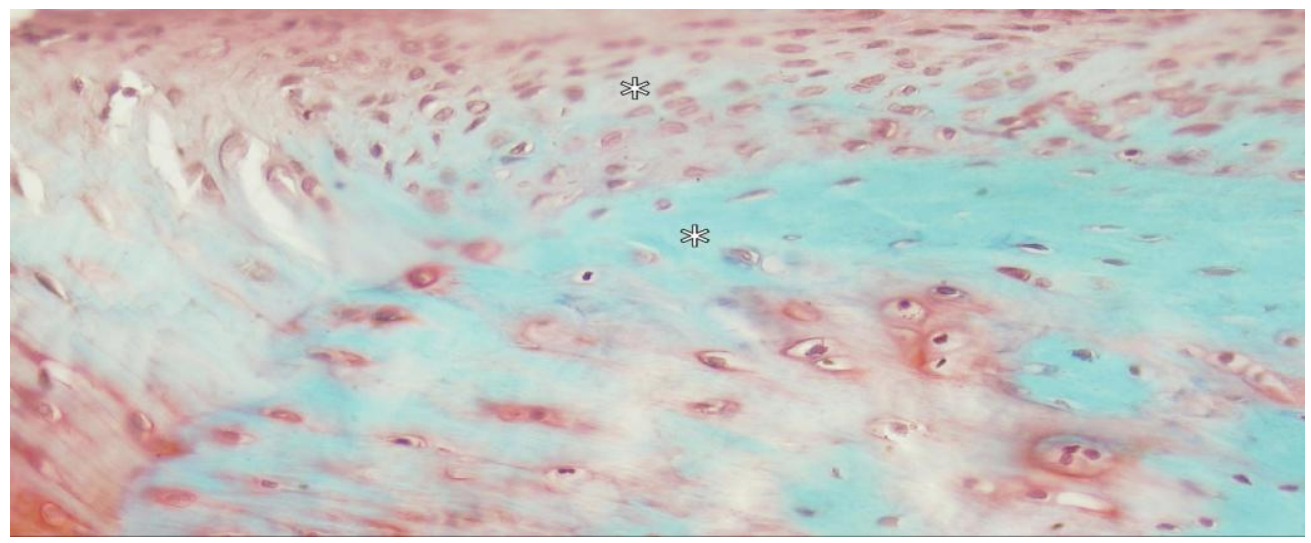

Figure (19): A high magnification of figure (18) showing marked decrease of the proteoglycan red staining (stars).

(Safranin $O$ / fast green $x$ 400)

B) Results of Image Analysis and Statistical Analysis:

\section{1- A comparison of the thickness ( $\mu \mathrm{m})$ of the hyaline} articular cartilage covering the central part of femoral condyle at a magnification $\times 40$ between 2 , 6 and 10 weeks in control group (I) and arthritis group (II):

\section{Using ANOVA test:}

As regard the control group (I), there was insignificant ( $\mathbf{P = 0 . 9 9 )}$ difference in the articular cartilage thickness at 2, 6 and 10 weeks from the onset of the experiment. However, in arthritis group (II), there was a significant $(\mathbf{P}<\mathbf{0 . 0 0 1})$ change noticed in the articular cartilage thickness after 2, 6 and 10 weeks from the onset of the experiment (Histogram 1).

\section{Post-hoc Tukey:}

As regard arthritis group (II), the articular cartilage thickness showed significant $(\mathbf{P}=\mathbf{0 . 0 2})$ decrease in rats sacrificed after 6 weeks compared to rats sacrificed after 2 weeks from the onset of the experiment. In addition, the articular cartilage thickness was significantly (P<0.001) decreased in rats sacrificed after 10 weeks compared to rats sacrificed after 2 weeks and significantly $(\mathbf{P}=\mathbf{0 . 0 1})$ decreased compared to rats sacrificed after 6 weeks from the onset of the experiment (Histogram 1). 


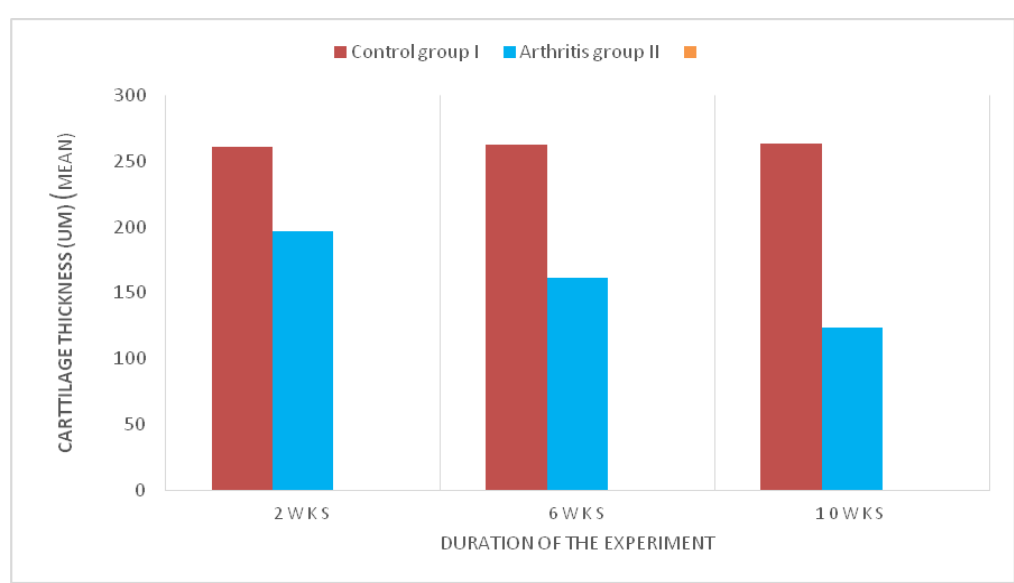

Histogram (1): column chart shows mean of articular cartilage thickness $(\mu \mathrm{m})$ of femoral condyle between 2 , 6 and 10 weeks in control group (I) and arthritis group (II)

\section{2- A comparison of the percentage $(\%)$ of the area} of collagen stained with Masson's trichrome stain in the articular cartilage covering femoral condyle at a magnification $x 100$ between 2,6 and 10 weeks in control group (I) and arthritis group (II):

\section{Using ANOVA test:}

As regard the control group (I), there was insignificant ( $\mathbf{P = \mathbf { 0 . 8 7 }})$ difference in collagen content in the articular cartilage represented by area stained by Masson's trichrome stain after 2, 6 and 10 weeks from the onset of the experiment. However, in arthritis group (II), there was significant $(\mathbf{P}<\mathbf{0 . 0 0 1})$ change noticed in collagen content at 2, 6 and 10 weeks from the onset of the experiment (Histogram 2).

\section{Post-hoc Tukey:}

As regard arthritis group (II), the collagen content of the articular cartilage represented by area stained by Masson's trichrome stain showed significant $(\mathbf{P}<\mathbf{0 . 0 0 1})$ decrease in rats sacrificed after 6 weeks compared to rats sacrificed after 2 weeks from the onset of the experiment. In addition, the collagen content was significantly (P $<\mathbf{0 . 0 0 1})$ decreased in rats sacrificed after 10 weeks compared to rats sacrificed after 2 weeks and 6 weeks from the onset of the experiment (Histogram 2).

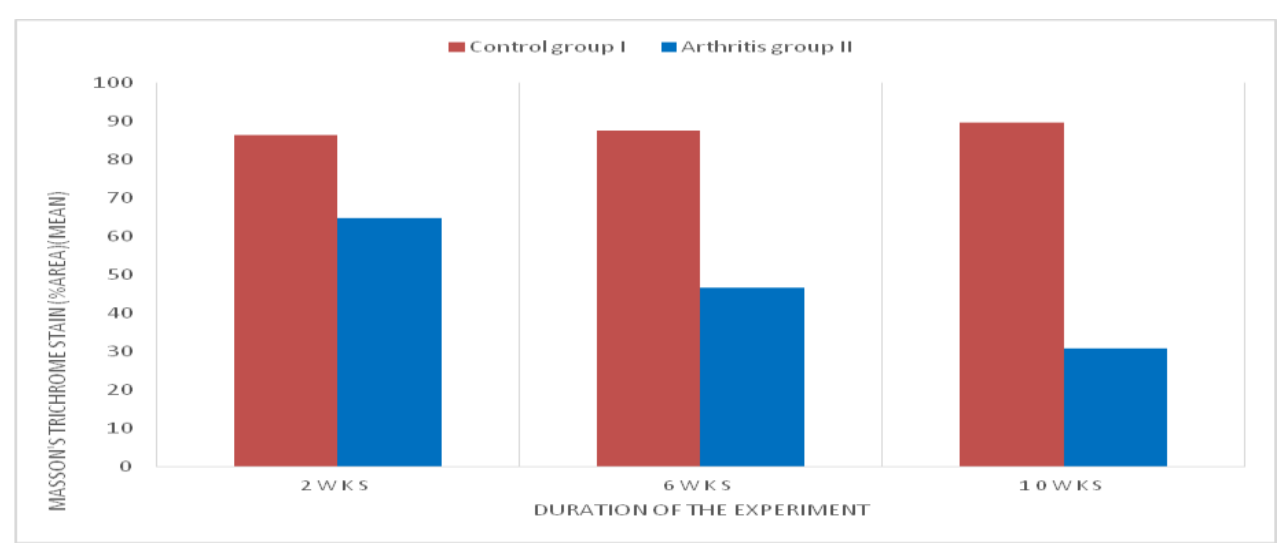

Histogram (2): column chart shows mean of the percentage (\%) of the area of collagen stained with Masson's trichrome stain between 2, 6 and 10 weeks in control group (I) and arthritis group (II) 
3- A comparison of the percentage $(\%)$ of area of proteoglycans stained with Safranin stain in the articular cartilage covering femoral condyle at a magnification $x 100$ between 2,6 and 10 weeks in control group (I) and arthritis group (II):

\section{Using ANOVA test:}

As regard the control group (I), there was insignificant $(\mathbf{P}=\mathbf{0 . 9 9})$ difference in proteoglycan content in the articular cartilage represented by area stained by safranin $O$ fast green stain at 2, 6 and 10 weeks from the onset of the experiment. However, in arthritis group (II), there was a significant ( $\mathbf{P}<\mathbf{0 . 0 0 1})$ change noticed in proteoglycan content at 2, 6 and 10 weeks from the onset of the experiment (Histogram 3).

\section{Post-hoc Tukey:}

As regard arthritis group (II), the proteoglycan content represented by area stained by safranin $\mathrm{O}$ fast green stain of the articular cartilage showed significant $(\mathbf{P}<\mathbf{0 . 0 0 1})$ decrease in rats sacrificed after 6 weeks compared to rats sacrificed after 2 weeks from the onset of the experiment. In addition, the proteoglycan content was significantly $(\mathbf{P}<\mathbf{0 . 0 0 1})$ decreased in rats sacrificed after 10 weeks compared to rats sacrificed after 2 weeks and 6 weeks from the onset of the experiment (Histogram 3).

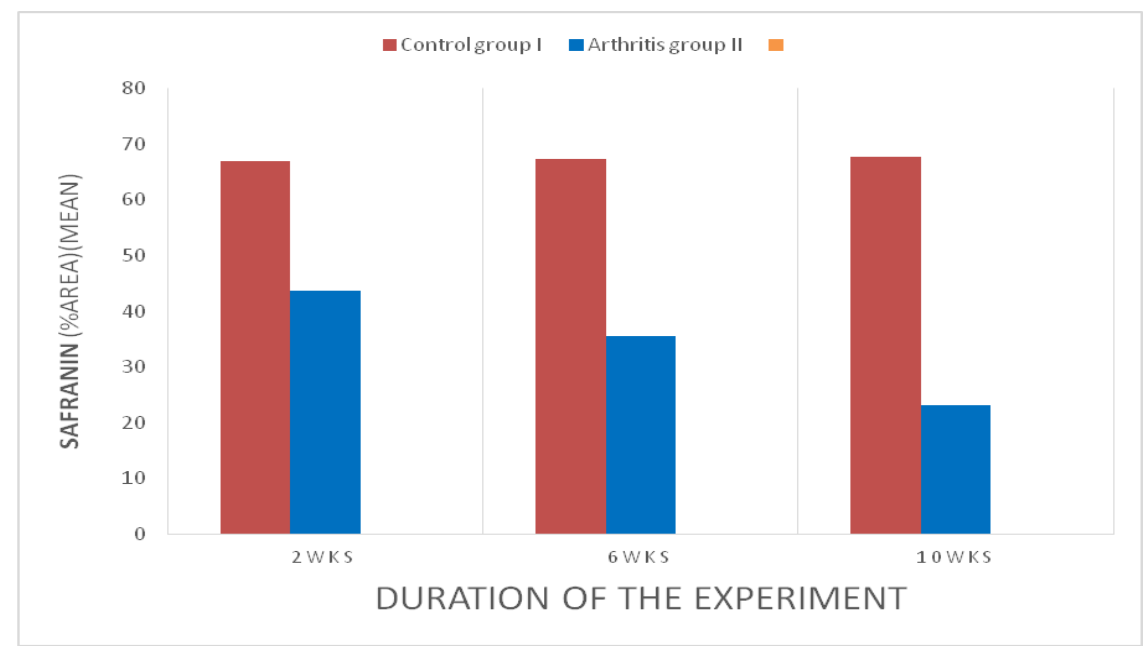

Histogram (3): column chart shows mean of the percentage (\%) of the area of proteoglycans stained with Safranin stain between 2, 6 and 10 weeks in control group (I) and arthritis group (II)

\section{Discussion:}

Arthritis is the most common joint disorder. The most significant structural change seen in arthritis is the degeneration of the articular cartilage. Several animal models have been developed to study the pathogenesis of arthritis and the efficacy of new diagnostic tools and therapeutic interventions. To date, there is no single ideal experimental model that permits investigation of all features of arthritis, and each model of induction has distinct advantages and disadvantages (Little \& Smith, 2008).
We generated a rat chronic arthritis model using complete Freund's adjuvant (CFA).CFA is inactivated dried mycobacteria which is responsible for stimulation of cell-mediated immunity that ultimately increased the production of certain immunoglobulins (Tuncel et al., 2016; Mahdi et al., 2018).

In the current study, control rat knee joint showed that the hyaline articular cartilage had a smooth surface, the matrix exhibited normal architecture and the cells were intact with appropriate orientation. The chondrocytes and 
surrounding matrix were arranged in well-ordered zones: the superficial zone, the middle zone, the deep zone and the calcified zone. The matrix of articular cartilage showed normal content of collagen and proteoglycans. These results were supported by Jeong et al. (2018), Salman et al. (2019) and Mohammed et al. (2018).

In the present study, the CFA-induced arthritis rat model showed time-dependent degeneration manner of the hyaline articular cartilage of the knee joint. There was progressive decrease in the thickness of articular cartilage. The chondrocytes and surrounding matrix exhibited severe disorganization. Chondrocytes showed degeneration, hypertrophy and extensive proliferation. Moreover, the matrix exhibited fibrillations, fissuring, delamination and denudation with reparative tissue "fibrous tissue" within the denuded surface. Statistically, the thickness of the hyaline articular cartilage was significantly decreased in arthritis group compared to control group. This agreed with $X u$ et al. (2016) who mentioned the changes of condylar cartilage in rat inflamed temporomandibular joint induced by CFA.

Moreover, the induced arthritis rat model revealed progressive decrease in collagen and proteoglycan content in the matrix of the articular cartilage compared to control sections. Matrix fissures were noticed with complete loss of collagen and proteoglycans. With progression of the disease, the articular cartilage was replaced by fibrous tissue which contained collagen fibers and multiple nuclei. Statistically, collagen and proteoglycan contents in the articular cartilage were significantly decreased in arthritis group. These results were supported by $\mathbf{L i}$ et $\mathbf{a l}$. (2016) and Hasan et al. (2018) who established a rat arthritis model by injecting CFA. They reported synovial hyperplasia, cellular infiltration, pannus formation (granulation tissue or fibrous tissue formation) and progressive decrease in proteoglycan and collagen content of the matrix.

Sugimoto et al. (2013) and Shuang et al. (2014) reported that complete Freund's adjuvant can non-specifically promote antigen sensitization process and improve immune response. As a result, many cell types such as macrophages, lymphocytes, synovial cells and neutrophils aggregate into the articular cavity, releasing mediators and inflammatory cytokines such as TNF- $\alpha$, IL-1, and IL-2. These released materials establish a complex niche that aggravates joint damage.

At first, compensatory mechanisms are able to maintain the integrity of the articular cartilage such as increased chondrocyte proliferation and clustering associated with increased synthesis of matrix molecules (collagen, proteoglycans (Mcinnes \& Schett, 2011).

As the disease progresses, there is evidence that the catabolic activity increases. The inflammatory cytokines bind to chondrocyte receptors inhibiting type II collagen production and stimulating the chondrocyte to release degradative enzymes including matrix metalloproteinase (MMP) 1, MMP3, MMP9, MMP13, MMP14, in addition to the aggrecanases [a disintegrin and metalloproteinase with thrombospondin-like motifs] ADAMTS4, ADAMTS5 and ADAMTS9 (Murphy \& Nagase, 2008; Stannus et al., 2010; Mei et al., 2017).

The disruption of chondrocyte homeostasis results in decreased proteoglycan content, 
increased water content of the extracellular matrix and weakening of the collagen network. Furthermore, there is increased apoptosis of chondrocytes (Loeser et al. 2012).

Degenerative changes in the articular cartilage lead to cartilage softening, fissuring, diminished cartilage thickness and formation of fibrillation zone of the superficial layers. With time, articular cartilage is totally destructed leaving the underlying subchondral bone plate exposed. In addition, the inflammatory cytokines enhance synthesis of collagen by synovial cells leading to formation of fibrous tissue at the site of excavated cartilage as an attempt of repair (Mcinnes \& Schett, 2011; Man \& Mologhianu, 2014).

According to our study, The CFA rat arthritis model resembles human degenerative arthritis in terms of the histopathological changes. Therefore, it can be very predictive in testing of the efficacy of novel anti- arthritic compounds. Our opinion is supported by Snekhalatha et al., 2013 who reported that adjuvant-induced arthritis is one of the best experimental models to study the effects of arthritis and is still widely used in the preclinical testing of rheumatoid arthritis. However, Samvelyan et al., 2021 reported that the validity of this model is questionable due to rapid and widespread cell death and joint changes atypical to human arthritis pathophysiology.

A potential limitation of this study must be considered: only a limited number of rats were used for this study as indicated by Institutional Review Board (IRB). It would provide better conclusions if validated with a larger sample size.

\section{Conclusion:}

In the present study, the CFA-induced arthritis rat model showed time-dependent degeneration manner of the hyaline articular cartilage of the knee joint. The chondrocytes showed degeneration, hypertrophy and extensive proliferation. Moreover, the matrix exhibited fibrillations, fissuring, delamination and denudation with fibrous tissue within the denuded surface. The thickness of the articular cartilage, the collagen content and the proteoglycan content showed significant decrease in rats sacrificed after 10 weeks compared to rats sacrificed after 2 weeks and 6 weeks from the onset of the experiment. This disease model can be used in testing of the efficacy of novel anti- arthritic compounds. However, more studies need to be conducted to validate this model for arthritis studies.

\section{References:}

1. Bancroft, J.D. and Layton, C. (2013): The hematoxylins and eosin \& Connective and mesenchymal tissues with their stains. In: In Survana, S.K., Layton, C. and Bancroft, J.D. (Eds.), Bancroft' Theory and Practice of Histological Techniques (7th Ed). Oxford, Churchill Livingstone Elsevier, 173- 213.

2. Evans C. H. (2013). Advances in regenerative orthopedics. Mayo Clinic proceedings, 88(11), 1323-1339.

3. Harrell, C. R., Markovic, B. S., Fellabaum, C., Arsenijevic, A., \& Volarevic, V. (2019). Mesenchymal stem cell-based therapy of osteoarthritis: Current knowledge and future perspectives. Biomedicine \& 
pharmacotherapy $=$ Biomedecine $\&$

pharmacotherapie, 109, 2318-2326.

4. Hasan, H., Ismail, H., El-Orfali, Y., \& Khawaja, G. (2018). Therapeutic benefits of Indole-3-Carbinol in adjuvant-induced arthritis and its protective effect against methotrexate induced-hepatic toxicity. BMC complementary and alternative medicine, 18(1), 1-12.

5. Hazra, A., \& Gogtay, N. (2016). Biostatistics series module 6: correlation and linear regression. Indian journal of dermatology, 61(6), 593.

6. Hirschmann, M. T., \& Müller, W. (2015). Complex function of the knee joint: the current understanding of the knee. Knee surgery, sports traumatology, arthroscopy: official journal of the ESSKA, 23(10), 2780-2788.

7. Hunter, D. J., \& Felson, D. T. (2006). Osteoarthritis. BMJ (Clinical research ed.), 332(7542), 639-642.

8. Jeong, J., Bae, K., Kim, S. G., Kwak, D., Moon, Y. J., Choi, C. H., Kim, Y. R., Na, C. S., \& Kim, S. J. (2018). Antiosteoarthritic effects of ChondroT in a rat model of collagenase-induced osteoarthritis. BMC complementary and alternative medicine, 18(1), 131.

9. Kahveci, Z., Minbay, F. Z., \& Cavusoglu, L. (2000). Safranin O staining using a microwave oven. Biotechnic \& histochemistry: official publication of the Biological Stain Commission, 75(6), 264268.

10. Li, C., Cai, H., Meng, Q., Feng, Y., Guo, H., Fang, W., \& Long, X. (2016). IL-1 $\beta$ mediating high mobility group box protein-1 expression in condylar chondrocyte during temporomandibular joint inflammation. Journal of Oral Pathology \& Medicine, 45(7), 539-545.

11. Little, C. B., \& Smith, M. M. (2008). Animal models of osteoarthritis. Current Rheumatology Reviews, 4(3), 175-182.

12. Liu, J. Y., Hou, Y. L., Cao, R., Qiu, H. X., Cheng, G. H., Tu, R., Wang, L., Zhang, J. L., \& Liu, D. (2017). Protodioscin ameliorates oxidative stress, inflammation and histology outcome in Complete Freund's adjuvant induced arthritis rats. Apoptosis : an international journal on programmed cell death, 22(11), 1454-1460.

13. Loeser, R. F., Olex, A. L., McNulty, M. A., Carlson, C. S., Callahan, M. F., Ferguson, C. M., Chou, J., Leng, X., Fetrow, J. S. (2012) Microarray analysis reveals age-related differences in gene expression during the development of arthritis in mice. Arthritis Rheum, 64(3), 705-717.

14. Mahdi, H. J., Khan, N., Asmawi, M., Mahmud, R., \& A/L Murugaiyah, V. (2018). In vivo anti-arthritic and antinociceptive effects of ethanol extract of Moringa oleifera leaves on complete Freund's adjuvant (CFA)-induced arthritis in rats. Integrative medicine research, 7(1), 85-94.

15. Malfait, A. M., Little, C. B., \& McDougall, J. J. (2013). A commentary on modelling osteoarthritis pain in small 
animals. Osteoarthritis

and

Cartilage, 21(9), 1316-1326.

16. Man, G. S., \& Mologhianu, G. (2014). Osteoarthritis pathogenesis - a complex process that involves the entire joint. Journal of medicine and life, 7(1), $37-41$.

17. Mcinnes, I. B., \& Schett, G. (2011). The pathogenesis of rheumatoid arthritis. New England Journal of Medicine, 365(23), 2205-2219.

18. Mei, L., Shen, B., Ling, P., Liu, S., Xue, J., Liu, F. \& Liu, X. (2017). Cultureexpanded allogenic adipose tissue-derived stem cells attenuate cartilage degeneration in an experimental rat osteoarthritis model. PLoS One, 12(4), e0176107.

19. Mohammed, S., Makhlouf, N., \& Baher, W. (2018). The Effect of Bone Marrow Mesenchymal Stem Cells Versus Methotrexate on the Knee Joint in A Rat Model of Rheumatoid Arthritis. A Histological Study. Journal of Medical Histology, 2(1), 45-56.

20. Murphy, G., \& Nagase, H. (2008). Reappraising metalloproteinases in rheumatoid arthritis and osteoarthritis: destruction or repair?. Nature clinical practice. Rheumatology, 4(3), 128-135.

21. Nickien, M., Heuijerjans, A., Ito, K., \& van Donkelaar, C. C. (2018). Comparison between in vitro and in vivo cartilage overloading studies based on a systematic literature review. Journal of Orthopaedic Research ${ }^{\circledR}, 36(8), \quad$ 20762086.
22. Ondrésik, M., Oliveira, J. M. \& Reis, R. L., (2016). Knee articular cartilage. In Oliveira JM and Reis RL (Eds.), Regenerative Strategies for the Treatment of Knee Joint Disabilities, Switzerland, Springer Nature, 3-20.

23. Pritzker, K. P., Gay, S., Jimenez, S. A., Ostergaard, K., Pelletier, J. P., Revell, P. A., Salter, D., \& van den Berg, W. B. (2006). Osteoarthritis cartilage histopathology: grading and staging. Osteoarthritis and cartilage, 14(1), 13-29.

24. Quasnichka, H. L., AndersonMacKenzie, J. M., \& Bailey, A. J. (2006). Subchondral bone and ligament changes precede cartilage degradation in guinea pig osteoarthritis. Biorheology, 43(3, 4), 389397.

25. Salman, A., Shabana, A. I., ElGhazouly, D. E., \& Maha, E. (2019). Protective effect of glucosamine and risedronate (alone or in combination) against osteoarthritic changes in rat experimental model of immobilized knee. Anatomy \& cell biology, 52(4), 498 510.

26. Samvelyan, H. J., Hughes, D., Stevens, C., \& Staines, K. A. (2020). Models of osteoarthritis: relevance and new insights. Calcified tissue international, 114.

27. Shuang, F., Zhu, J., Song, K., Hou, S., Liu, Y., Zhang, C., \& Tang, J. (2014). Establishment of a rat model of adjuvantinduced osteoarthritis of the lumbar facet 
joint. Cell biochemistry and

biophysics, 70(3), 1545-1551.

28. Snekhalatha, U., Anburajan, M., Venkatraman, B., \& Menaka, M. (2013). Evaluation of complete Freund's adjuvant-induced arthritis in a Wistar rat model. Zeitschrift für

Rheumatologie, 72(4), 375-382.

29. Stannus, O., Jones, G., Cicuttini, F., Parameswaran, V., Quinn, S., Burgess, J., \& Ding, C. (2010). Circulating levels of IL-6 and TNF- $\alpha$ are associated with knee radiographic osteoarthritis and knee cartilage loss in older adults. Osteoarthritis and cartilage, 18(11), 1441-1447.

30. Sugimoto, Y., Kojima, Y., Inayoshi, A., Inoue, K., Miura-Kusaka, H., Mori, K. \& Suzuki, N. (2013). K-685, a TRPV1 antagonist, blocks PKC-sensitized TRPV1 activation and improves the inflammatory pain in a rat complete Freund's adjuvant model. Journal of pharmacological sciences, 123(3), 256-266.

31. Tuncel, J., Haag, S., Hoffmann, M. H., Yau, A. C., Hultqvist, M., Olofsson, P., Bäcklund, J., Nandakumar, K. S., Weidner, D., Fischer, A., Leichsenring, A., Lange, F., Haase, C., Lu, S., Gulko, P. S., Steiner, G., \& Holmdahl, R. (2016). Animal Models of Rheumatoid Arthritis (I): Pristane-Induced Arthritis in the Rat. PloS one, 11(5), e0155936.
32. Valentim, A. M., Guedes, S. R., Pereira,
A. M., \& Antunes, L. M. (2016).
Euthanasia using gaseous agents in laboratory rodents. Laboratory animals, 50(4), 241-253.

33. Wu Y. (2015). Introduction. In Knee Joint Vibroarthrographic Signal Processing and Analysis, New York, Springer, 1-8.

34. Xu, L., Guo, H., Li, C., Xu, J., Fang, W., \& Long, X. (2016). A time-dependent degeneration manner of condyle in rat CFA-induced inflamed TMJ.American journal of translational research, 8(2), 556-567. 